\title{
Gestione avanzata delle risorse naturali con i sistemi acquaponici: analisi tecnica ed economica
}

\author{
Luca Lombardo $^{1}$ and Domenico Giaquinto ${ }^{1}$
}

\section{${ }^{1}$ Affiliation not available}

\begin{abstract}
L'innalzamento delle temperature e la riduzione delle precipitazioni influiranno col passare del tempo sulla produttività agricola con un impatto pressoché negativo, inoltre, la non sostenibilità di alcune pratiche agricole con il riversamento degli scarti prodotti determina un concreto inquinamento che mette in serio rischio l'ecosistema naturale. Una delle soluzioni a queste tematiche potrebbe essere l'acquaponica, producendo cibo di qualità in maniera sostenibile e competitiva, un sistema che garantisce una maggiore resa e una maggiore velocita di crescita delle piante coltivate. Dunque, creare un ecosistema naturale in un ambiente artificiale produce uno spreco minimo, richiede input energetici limitati e produce impatti postivi sull'ambiente. In tali impianti l'acqua circola dalle vasche dei pesci raggiungendo e fertilizzando le piante, per tornare poi "depurata" nella vasca dei pesci pronta a eseguire nuovamente lo stesso ciclo. Questo consente di recuperare tutta l'acqua che le piante non sono state in grado di assorbire, riducendo del $90 \%$ il consumo acqua per kg di prodotto rispetto all'agricoltura tradizionale. Il seguente articolo intende valutare la fattibilità tecnica-economica di un sistema acquaponico mediante la realizzazione di un progetto preliminare di un'area ricadente nel comune di Alfano (SA). Il sistema progettato occupa una superficie netta di $1075 \mathrm{~m} 2$ di cui $36,6 \%$ di acquacoltura, $46,2 \%$ coltivazione piante e $17,2 \%$ vasche di raccolta; per una gestione efficiente dell'impianto sono previsti una serie di trattamenti: filtro meccanico a tamburo, filtrazione biologica e trattamento lampada UV. Infine, a seguito dell' analisi costi benefici effettuata è stato stimato un tempo di ritorno semplice dell'investimento pari a 3 anni e mezzo.
\end{abstract}




\section{Introduzione}

C'è un crescente interesse per l'acquaponica tra le imprese sociali, perché rappresenta uno strumento efficace per aiutarli a svolgere il proprio mandato. Per esempio, può integrare strategie di sostentamento per garantire cibo e piccoli redditi per famiglie povere e senza terra. Produzione interna di cibo, accesso a mercati e l'acquisizione di competenze sono strumenti di inestimabile valore per garantire un futuro nei paesi in via di sviluppo, e l'acquaponica può fornire le basi per una crescita socioeconomica equa e sostenibile ${ }^{1}$. Essa mira a ridurre gli impatti abientali e a ridurre al minimo l'inquinamento (ad esempio, le acque reflue) ${ }^{2}$ massimizzando la produzione efficienza e stabilità, aumentando quindi i ricavi ${ }^{3}$. Ad esempio, rispetto all'agricoltura convenzionale, utilizza meno del 10\% di acqua, a seconda delle condizioni climatiche ${ }^{4}$. Circa l'1-3\% del volume totale di acqua al giorno deve essere reintegrato ${ }^{5}$. L'acquaponica comporta anche un uso inferiore di fertilizzante rispetto a quello convenzionale o sistema idroponico ${ }^{6}$. Al giorno d'oggi, può essere considerata una tecnologia emergente e in via di sviluppo argomento scientifico ${ }^{7}$ che contribuisce a una maggiore sostenibilità sistemi alimentari ${ }^{8}$ in diversi contesti - urbano / rurale, su piccola / grande scala, paesi sviluppati / in via di sviluppo ${ }^{9}$. L'acquaponica mette insieme coltivazione idroponica e acquacoltura. L'impianto riesce a creare un ecosistema chiuso e circolare: l'ammoniaca presente negli escrementi dei pesci viene trasformata da alcuni particolari batteri per diventare nutrimento delle piante, che a loro volta purificano l'acqua per i pesci. Un processo naturale, quindi, può essere definito come una tecnica sinergica che combina la produzione simultanea di piante (coltura idroponica), pesce (acquacoltura) e batteri, per scopi commerciali, domestici, ornamentali o educativi. I principi su cui si basa il sistema sono i seguenti: l'acquacoltura consuma grandi quantità di acqua che viene espulsa perché satura di composti azotati derivanti dalle deiezioni dei pesci che la rendono tossica; la coltura idroponica consuma grandi quantità di ferti- 
lizzanti che vengono miscelati nella soluzione circolante. Quindi nell'acquaponica, l'acqua esegue due funzioni fondamentali: ospita i pesci e consente la crescita delle piante, quindi l'acqua viaggia attraverso le radici delle piante, esse assorbono i nutrienti e infine l'acqua depurata ritorna alla vasca del pesce. Questo processo permette a pesci, piante e batteri di prosperare in simbiosi e di lavorare insieme per creare un ambiente di crescita sano per gli uni e per gli altri, a condizione che il sistema sia correttamente bilanciato. Le piante migliorano la loro crescita usando il metabolico rifiuto di pesce e mangimi non consumati, che vengono trasformati da a comunità batterica in nutrienti facilmente assimilabili (ad es. nitrati, fosfati). A causa dell'estrazione effettuata dal sistema radicale delle piante, l'acqua viene purificata da nitrati e fosfati eccessivi, mantenendo livelli adeguati allo sviluppo dei pesci ${ }^{10}$. Vanno inoltre valutate e controllate le emissione odorigine che sono presenti negli impianti acquaponici .

\section{Progettazione impianto acquaponico}

In base ai punti di forza e di debolezza si è deciso di studiare la fattibilità tecnica-economica di un impianto acquaponico Floating, con base DWC ma con la particolarità delle zattere galleggianti. Dunque, le radici nude delle piante saranno immerse completamente all'interno dell'acqua contenente sostanza nutritiva.

La scelta del luogo dove installare l'impianto è un aspetto molto importante. L'impianto in oggetto deve essere realizzato in un terreno di $336 \mathrm{~m}$ di altitudine e $9500 \mathrm{~m} 2$ di superficie, ubicato nella località di Alfano provincia di Salerno. L'impianto sfrutta $1075 \mathrm{~m} 2$ rispetto ai $9500 \mathrm{~m} 2$ disponibili dei quali 393,70 m2 sono destinati all'allevamento della specie ittica scelta, che è il persico trota, i restanti $681.30 \mathrm{~m} 2$ sono destinati alla coltivazione delle piante e la raccolta delle acque. Per quanto riguarda le vasche di allevamento del persico trota, sono 4 da $25 \mathrm{~m} 3$ di forma circolare contenente 25.000 litri a vasca per un totale di 100.000 litri, la quantità massima di allevamento è di 1750 pesci. Pertanto, tali vasche saranno collegate tramite tubazione ad un filtro meccanico 
a tamburo che svolgerà un ruolo di fondamentale importanza per l'equilibrio dell'intero sistema (Fig.2). Il suo funzionamento ottimizzato permette la pulizia automatica della rete di filtrazione meccanica, quando essa sarà completamente sporca il livello dell' acqua diminuirà, tale condizione farà azionare un sensore che farà avviare la pulizia del rullo grazie ad una pompetta che alimenterà degli ugelli che spruzzeranno acqua sul rullo a rete. In questo modo lo sporco si depositerà in un'apposita canaletta sottostante che lo farà defluire fuori dal filtro per essere smaltito. Oltre al sistema di filtrazione è stato previsto un trattamento lampada UV con azione alghicida, battericida e virucida. Dalla componente di filtrazione, l'acqua tramite pompa in acciaio passa alle vasche per la coltivazione delle piante in telo PVC, sono anch'esse 4, da 20x5m e $0.25 \mathrm{~m}$ di profondità con una superficie coltivabile di $400 \mathrm{~m} 2$. All'interno saranno collocati degli ossigenatori per garantire la giusta quantità di DO (ossigeno disciolto), sopra queste vasche vanno posizionati 520 pannelli galleggianti forati con vasetti contenenti le piante. Le piante coltivabili sono circa 60.000 all'anno ma molto dipende da fattori come l'accurata gestione dell'impianto, dai fattori climatici, il rispetto della biosicurezza in impianto e la domanda dei prodotti sul mercato.

Dopo aver alimentato le piante, l'acqua verrà raccolta in apposite vasche (4 da $5 \times 3 \mathrm{~m})$ per poi essere incanalate in un'unica tubazione ed essere rispedita tramite pompa nelle vasche dei pesci;

Nell'impianto è previsto lo Smart System, ovvero, sistema intelligente che incorpora funzioni di rilevamento, attuazione e controllo al fine di descrivere e analizzare una situazione e prendere decisioni basate sui dati disponibili in modo predittivo o adattivo, eseguendo quindi azioni intelligenti. Per esempio, il sistema di areazione e ricircolo si raddoppiano in situazioni di emergenza, inoltre, è prevista una sonda per il monitoraggio dell'ossigeno per ogni vasca di allevamento e sensori di pH e temperatura per tutte le vasche dell'impianto, il tutto collegato ad apposito allarme. Infine, è previsto il cosiddetto Fog system, un sistema di raffreddamento della serra dedicata alla coltivazione delle piante. L'alto livello di tecnologia delle sonde e i test dei parametri chimico-fisici ci 
permettono di garantire un' accurata gestione dell'impianto.

La potenza impegnata sarà pari ad un massimo di circa $6 \mathrm{~kW}$, con un impianto elettrico monofase e nel caso in cui ci fossero delle interruzioni per cause esterne è stato previsto un generatore di emergenza da $5 \mathrm{~kW}$ a diesel per sopperire tale mancanza.

\section{Costi di investimento e costi di gestione}

I costi di investimento includono tutti gli elementi necessari alla progettazione e alla realizzazione dell'impianto acquaponico che produce piante e pesci a scopo produttivo (vedi tabella).

Altri elementi:

- Giunti e rubinetteria

- Strumenti di gestione impianto (sonde, test parametri chimico-fisici, guadini)

- Tubazioni

Le singole voci dei costi di gestione (IVA esclusa) sono state analizzate tramite informazioni procurate da un'analisi di mercato intervistando le aziende interessate. Il persico trota viene venduto da 0.45 ad avannotto per acquisti da i 1000 esemplari in su, è un pesce carnivoro e ciò implica che i mangimi ad esso dedicato sono molto proteici e vengono prodotti da circa 10 aziende presenti sul territorio nazionale con prezzo da $1.27 / \mathrm{Kg}$, ogni pesce lungo il suo percorso di allevamento mangia circa $800 \mathrm{~g}$ totali per arrivare ad una taglia media di vendita che si aggira tra i 350/400 g. Per quanto riguarda le piante si è deciso di coltivare insalata per il 60\% della capacità del sistema con 36.000 pezzi e piante officinali per il restante $40 \%$ con 24.000 pezzi. Il costo delle piantine da trapiantare è stato ricavato tramite indagine sul mercato con un costo di 0.04 al pezzo e le piante 
officinali a 0.08 al pezzo. In particolare, le piante officinali verranno vendute ad un certo punto della crescita in vasetti $12 \mathrm{~cm}$ di diametro e $10 \mathrm{~cm}$ di altezza riempiti con $10 \mathrm{~cm} 3$ di terriccio che equivale ad 1 litro.Le spese relative alla corrente elettrica ed acqua sono state stimate in base a tariffe standard con relativa distinzione tra quote fisse e quote variabili. L'impianto non ha bisogno di manutenzione ordinaria, essendo progettato con elementi all'avanguardia garantendo almeno 5 anni di ottimo funzionamento, dopo il quale c'è l'eventuale possibilità di dover effettuare una manutenzione straordinaria dovuta dall'usura degli stessi. La manodopera necessaria per tale impianto è di due persone per un tempo giornaliero che dipende dai periodi di coltivazione delle piante, pertanto, una volta messe a dimora tutte le unità predisposte, dove, un compito giornaliero essenziale, sarà quello di nutrire la specie ittica allevata. La voce del marketing verrà curata da un esperto del settore, che con tecniche aggiornate e promozioni darà luogo ad una campagna pubblicitaria per la vendita dei prodotti. In particolare, gestirà: creazione sito web, logo, ottimizzazione SEO (Search Engine Optimization), promozioni social e gestione. La voce altre spese sono state stimate per circa il 20\% dei costi di gestione annui e includono: oneri finanziari, manutenzione ordinaria e straordinaria, imprevisti e locazione spazi deposito.

I ricavi come per i costi di gestione sono stati valutati su base annuale.

Dunque, le piante officinali sarà ciò che creerà maggiore redditività con il 61,5\% sul ricavo totale, poi l'insalata con il $31,4 \%$ e infine, il persico trota con il $7,1 \%$. Da queste percentuali si nota palesemente che la redditività di tale impianto è data principalmente dalla coltivazione delle piante, invece, il pesce sarà e dovrà essere un'entrata secondaria. 


\section{Conclusione e discussione finale}

Dal punto di vista economico abbiamo un costo di investimento netto IVA di $115.000,00$ e nell' arco della gestione a dei ricavi di 97.600,00 /anno si contrappongono dei costi di 64.613,07 /anno. Quindi , nell'arco dell'anno si genera un flusso di cassa consistente di 32.986,93 /anno, che, sostanzialmente consente di recuperare i costi di investimento in circa 3 anni e mezzo.

Si è analizzata la fattibilità tecnica-economica per la realizzazione di un impianto acquaponico ad Alfano in provincia di Salerno. In base alle indagini eseguite sul territorio nazionale, il quale, hanno fornito notevoli informazioni, costi di investimento, costi di gestione e l'analisi dei prezzi, con importi netto IVA, si è arrivati alla conclusione che nonostante la realizzazione di un impianto acquaponico ha dei costi di investimento significativi, tale tecnica è redditizia, con un flusso di cassa pari a 32.986,93, con il quale si riescono a coprire le spese dei costi di investimento in circa 3 anni e mezzo. Pertanto, in tale impianto nessuna unità coltivabile è fissa, la coltivazione può variare in base al mercato. Infatti, se ci fosse più richiesta sul mercato di piante officinali, aumentando la quantità di coltivazione di tale unità e diminuendo l'insalata, il ricavo annuale dell'azienda aumenterà significativamente, complice il maggior prezzo di vendita rispetto all'insalata. Ciò genererà anche un aumento del flusso di cassa annuale, garantendo un notevole incremento della redditività dell'impianto. Come evince da tale analisi il fatturato principale verrà dalle piante, in quanto, il ricavo previsto dal persico trota sarà pari solo al 7,1\% del ricavo totale, dunque, avere un mercato vicino con la massima domanda e buoni prezzi è la prima condizione per il successo. Sfruttare tramite il marketing tutti i vantaggi dell'acquaponica rispetto alle pratiche tradizionali e far comprendere l'importanza dell'economia circolare, della sostenibilità della tecnica e l'alta qualità di 
un prodotto, che viene fuori da un processo naturale, senza l'utilizzo di pesticidi e fertilizzanti, genererà un incremento del mercato.

\section{References}

1.Somerville, C., Cohen, M., Pantanella, E., Stankus, A. \& Lovatelli, A. . Small-scale Aquaponic Food Production: Integrated Fish and Plant Farming. ., Food and Agriculture Organization of the United Nations (2014).

2.Blidariu, F. \& Grozea, A. . Increasing the economical efficiency and sustainability of indoor fish farming by means of aquaponics.. Anim. Sci. Biotechnol. (2011).

3.Tyson, R. V., Treadwell, D. D. \& Simonne, E. H. Opportunities and challenges to sustainability in aquaponic systems.. Hortechnology (2011).

4.Bernstein, S. Aquaponic Gardening: A Step-By-Step Guide to Raising Vegetables and Fish Together.. New Society Publishers (2011).

5.Somerville, C., Cohen, M., Pantanella, E., Stankus, A. \& Lovatelli, A. Small-scale Aquaponic Food Production: Integrated Fish and Plant Farming. Food and Agriculture Organization of the United Nations (2014).

6.Tyson, R., Hochmuth, R. \& Cantliffe, D. J. Hydroponic Vegetable Production in Florida. (2009).

7.König, B., Janker, J., Reinhardt, T., Villarroel, M. \& Junge, R. Analysis of aquaponics as an emerging technological innovation system. (2018).

8.Goddek, S. et al. . Challenges of sustainable and commercial aquaponics. (2015).

9.Strategic points in aquaponics. (2017).

10.Rakocy, J. E., Masser, M. P. \& Losordo, T. M. Recirculating aquaculture tank production sys- 
tems: aquaponics- integrating fish and plant culture.. (2006). 


\section{Figure Captions}

Figure 1. Floating system

Figure 2. Funzionamento impianto acquaponico

Figure 3. Progetto preliminare impianto acquaponico

Figure 4. Componenti dell'impianto

Figure 5. Costo totale di investimento

Figure 6. Costi di gestione annuale

Figure 7. Ricavi annui in gestione

Figure 8. Costi di investimento

Figure 9. Ricavi e costi in gestione

Figure 10. Flussi di cassa 
Figures

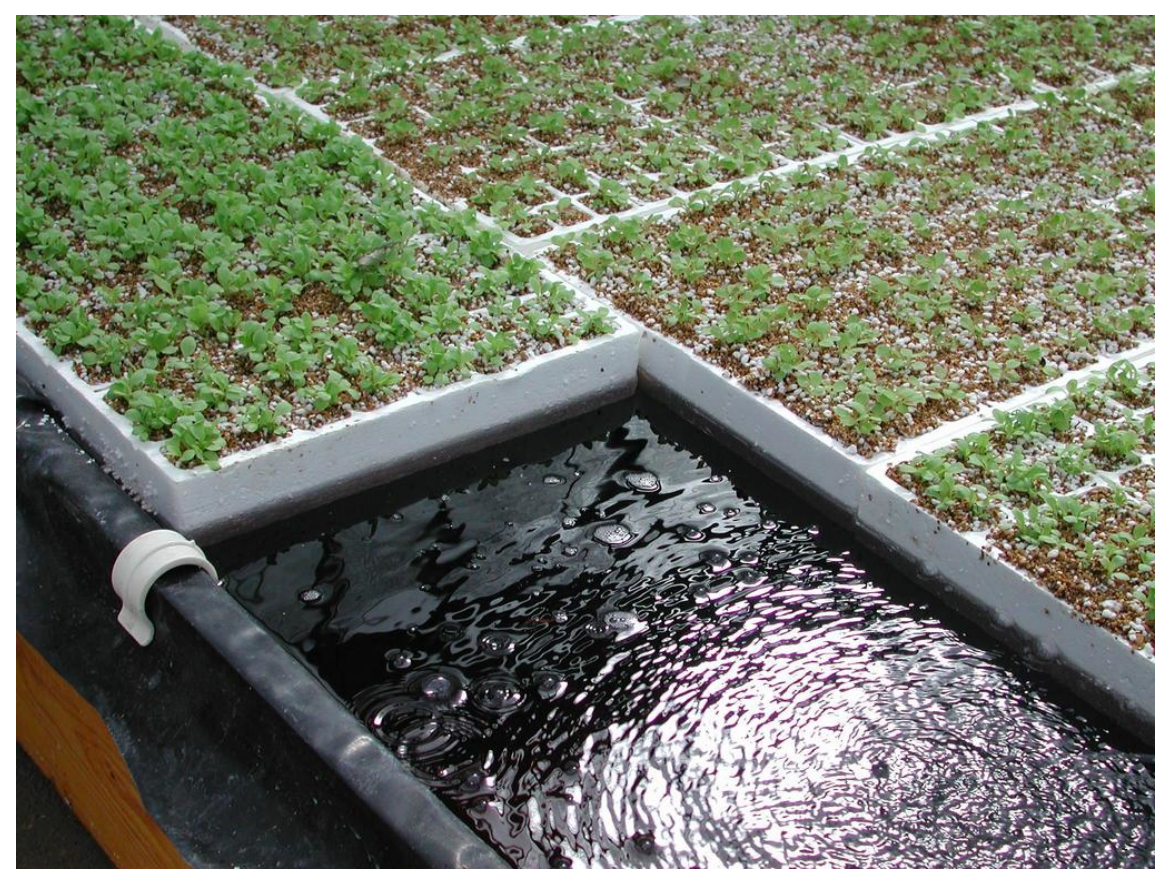

Figure 1: Floating system 


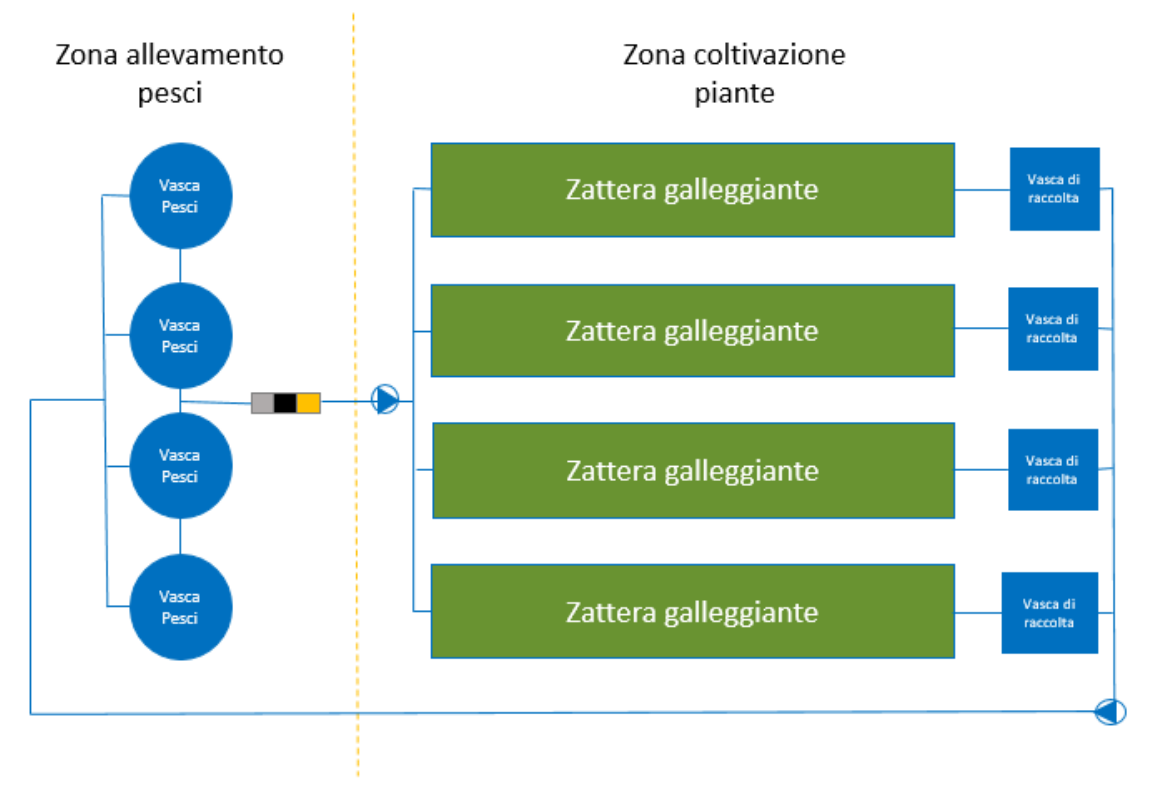
LEGENDA:
$\square$ = Trattamento UV $\square=$ Filtro a tamburo
= Filtro biologico
$=$ Tubi irrigazione $\vartheta=$ Pompa

Figure 2: Funzionamento impianto acquaponico 


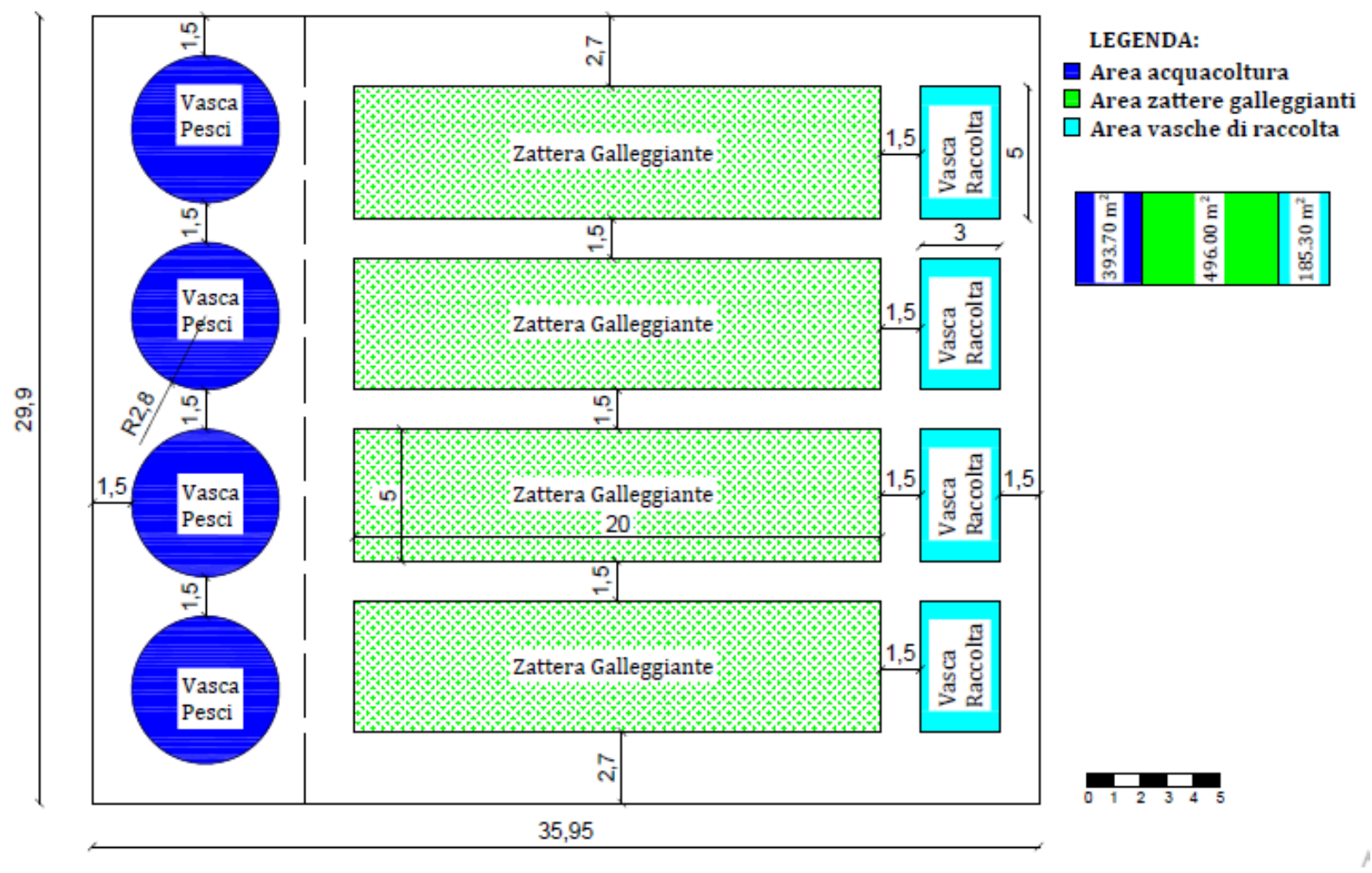

Figure 3: Progetto preliminare impianto acquaponico 


\begin{tabular}{|c|c|}
\hline COMPONENTI & QUANTITA' \\
\hline $\begin{array}{c}\text { Vasche con telo PVC per coltivazione } \\
\text { piante da } 25 \mathrm{~m}^{3}\end{array}$ & 4 \\
\hline $\begin{array}{c}\text { Pannelli galleggianti forati } \\
\text { Sistema di raffreddamento della serra } \\
\text { di coltivazione }\end{array}$ & 520 \\
\hline $\begin{array}{c}\text { Vasche allevamento ittico in PVC da } \\
25 \mathrm{~m}^{3}\end{array}$ & 1 \\
\hline $\begin{array}{c}\text { Vasche di raccolta acqua } \\
\text { Pompe in acciaio per la ricircolazione } \\
\text { dell'acqua (2 di riserva) }\end{array}$ & 4 \\
\hline $\begin{array}{c}\text { Lampada UV con azione alghicida, } \\
\text { battericida, viricida }\end{array}$ & 4 \\
\hline $\begin{array}{c}\text { Filtro meccanico a tamburo } \\
\text { Ossigenatori }\end{array}$ & 4 \\
\hline $\begin{array}{c}\text { Impianto elettrico monofase } \\
\text { Generatore di emergenza } 5 \mathrm{kw}\end{array}$ & 1 \\
\hline
\end{tabular}

Figure 4: Componenti dell'impianto

Figure 5: Costo totale di investimento 


\begin{tabular}{|c|c|c|c|c|}
\hline VOCI DI SPESA & UNITA' & QUANTITA' & $€ / \mathrm{UNITA}^{\prime}$ & $\begin{array}{c}\text { COSTO TOTALE } \\
€ / \text { anno }\end{array}$ \\
\hline Energia elettrica & Kwh & 52.560 & 0,16 & $8.409,60$ \\
\hline Acqua & $\mathrm{m}^{3}$ & 730 & 1,72 & $1.297,97$ \\
\hline $\begin{array}{l}\text { Mangime per } \\
\text { pesci }\end{array}$ & $\mathrm{Kg}$ & 1.400 & 1,27 & $1.778,00$ \\
\hline $\begin{array}{c}\text { Trapianto } \\
\text { piantine insalata }\end{array}$ & Pezzo & 36.000 & 0,04 & $1.440,00$ \\
\hline $\begin{array}{l}\text { Trapianto piante } \\
\text { officinali }\end{array}$ & Pezzo & 24.000 & 0,08 & $1.920,00$ \\
\hline Persico trota & Pezzo & 1750 & 0,45 & 787,50 \\
\hline Vasetti & Pezzo & 24.000 & 0,10 & $2.400,00$ \\
\hline Terriccio & $1000 \mathrm{~L}$ & 24 & 40,00 & 960,00 \\
\hline Manodopera & Mese & 1 & $1.850,00$ & $24.050,00$ \\
\hline Marketing & Mese & 1 & 900,00 & $10.800,00$ \\
\hline \multirow[t]{2}{*}{ Altre spese } & - & - & - & $10.770,00$ \\
\hline & & & & $64.613,07$ \\
\hline
\end{tabular}

Figure 6: Costi di gestione annuale

\begin{tabular}{|c|c|c|c|c|}
\hline VOCI DI RICAVO & UNITA' & QUANTITA' & ध/UNITA' & $\begin{array}{c}\text { TOTALE } \\
\text { ध/anno }\end{array}$ \\
\hline $\begin{array}{c}\text { Insalata } \\
\begin{array}{c}\text { Piante } \\
\text { officinali }\end{array}\end{array}$ & Pezzo & 36.000 & 0,85 & $30.600,00$ \\
\hline $\begin{array}{c}\text { Persico trota } \\
\text { Pezzo }\end{array}$ & $\mathrm{Kg}$ & 74.000 & 2,50 & $60.000,00$ \\
\hline
\end{tabular}

Figure 7: Ricavi annui in gestione

\section{Totale costi di investimento}

$115.000,00 €$

Figure 8: Costi di investimento 


\section{Ricavi in gestione \\ Costi di gestione \\ 97.600,00 €/anno \\ 64.613,07 €/anno}

Figure 9: Ricavi e costi in gestione

\section{Flussi di cassa}

$32.986,93 € /$ anno

Figure 10: Flussi di cassa 